\title{
Development of Robotic Rover with Controller \& Vision System
}

\author{
Roshahliza M Ramli ${ }^{1}$, Nurul Aqilah Herman ${ }^{2}$, Muhammad Ridzuan Mazlan ${ }^{3}$, \\ Azlin Suraya Nazaruddin', Muhammad Arshad Tuah", Abdul Naqib Eizzaz Abd Samad ${ }^{6}$, \\ Kamal Othman ${ }^{7}$, Yanti Marliana Esmail ${ }^{8}$ \\ 1,2,3,4,5,6 College of Engineering Technology, Universiti Malaysia Pahang, Malaysia \\ ${ }^{7,8}$ German Malaysian Institute, Malaysia
}

\begin{tabular}{l} 
Article Info \\
\hline Article history: \\
Received Sep 12, 2019 \\
Revised Nov 13, 2019 \\
Accepted Nov 27, 2019 \\
\hline
\end{tabular}

\section{Keywords:}

Robotic arm

Robotics

Rover

Search and rescue robots

Visual-based navigation

\begin{abstract}
Rover is a robotic system that integrates a simple system implementing electrical and mechanical components together. In this study, we propose a rover using mechanical components which consist of a robotic arm, joint and mechanical gripper, backbone chassis and continues track, while the electrical components include servo motor, servo controller, transmitter and receiver for vision system and wireless controller via USB host as its control system. The purpose of this project is for monitoring and safety purposes. In addition, the main goal of this project is to develop a simple robotic rover that is easy to build and manufacture as well as cost-effective. To add more functionality on this rover, it is equipped with a robotic arm and real-time view camera integration. This rover is equipped with a first-person view (FPV) camera, an integrated camera on the rover that can give clear visibility and direction to the rover pilot. The live feed can be viewed on the monitor inside the command station box. It can be used to assist safety authorities to collect information \& insights, work lift to collect and remove the load and to conduct search and rescue operation. As for the result, the mobility system of the robotic rover at terrain surfaces and analyses the capabilities of the chassis during lifting load had been tested.
\end{abstract}

Copyright (C) 2020 Institute of Advanced Engineering and Science. All rights reserved.

\section{Corresponding Author:}

Roshahliza M Ramli,

Faculty of Electrical \& Electronics Engineering Technology,

Universiti Malaysia Pahang,

26600 Pekan, Pahang Darul Makmur, Malaysia.

Email: roshahliza@ump.edu.my

\section{INTRODUCTION}

Rover has vast and wide applications in the real world. It has been implemented in space exploration, safety and weapon system endeavor, intelligent home application as well as military application $[1,2]$. In addition, a small and agile rover prototype such as The Light Weight Rover Unit (LRU) had existed for planetary exploration [3, 4]. After the fruitful robotic missions on Moon and Mars, tentative arrangements have been made for conducting different experiments and activities utilizing a robotic rover $[5,6]$. In this paper, the focus in the robotic rover system is for safety monitoring [7] where authority bodies such as Police Department, Hazard Material (HAZMAT) unit and Chemical Biological Radiation Nuclear explosive (CBRNE) unit may benefit from this work.

Robotic rover goes where humans fear to tread. Whether in a hazardous and dangerous condition, a robotic rover system can be applied to assess, reduce or eliminate the risk. Through this, the rover can prevent any life-threatening or risk of death on people that lurks with every wrong move. In the year 2003, a military colonel told the Associated Press that it was unendingly desirable over the need to supplant a robot than to lose an officer to a bomb or snare [8]. This prototype, which is called Safety Rover, can be used by all 
authority bodies such as Police and Fire Department. Nowadays, they need technology equipment as safety precautions for them to handle a dangerous situation.

Recently, the rover used robotic skills with capabilities in all complex and autonomously performed operations. This kind of robots as assistants is effectively pursued in different scholastic and business research tasks, for example, Fire Fighter Robot, Object Tracker \& Follower Robot, Fire Fighter Robot with Night Vision Camera and many more [9-11]. Previously developed rover, especially for space exploration, is expensive and the researchers need to employ specific software like VERVE, an integrated 3D view built [12] to assist in navigation.

Moreover, to shield against the rover being lost or coincidentally colliding with unforeseen impediments, researchers have established software to enable the rover to settle on its own safety decisions and to create results naturally without these confusing software [13]. The rover is equipped with a vision system by applying a drone camera, so the rover does not need specific software to monitor its conditions. It just needs to monitor safety more easily by using the vision system via the remote camera in First Person View (FPV) recording video or taking photos, manages the zoom new, or turn on and off flash torch at any time and most importantly, the rover can let us know the relative position and manage to obtain information $[14,15]$.

Mostly, the robotic rover is large, bulky and takes a lot of space to store. Space missions acquire gigantic presence of robots, where they can be allocated to perform essential activities under no human supervision [16]. Hence, our goal is to utilize a portable, small and light rover that can also perform rescue operations effectively with a vision system, robot arm, and gripper for safety purposes.

During dangerous and hazardous conditions, the rover can perform activities such as bomb threats, counter-terrorism and simple monitoring for collecting insights. Our rover also has an arm that can move in longitudinal motion with a gripper at the edge of the arm to remove the load. The robotic rover can be deployed for risk assessments thus preventing any injuries, life threat or even death that lurks upon people with every wrong move made. Besides, through robotic rover, search and rescue operations can be enhanced on the ground. Rough and complex terrains would then be able to be included in the capacity with respect to the designs of such robots [17-20]. Thus, that made us choose the continuous track to overcome the terrain difficulties.

\section{PROPOSED ROBOTIC ROVER}

In this section, the proposed robotic rover is explained, from its overall design as well as its locomotion, the remote controller and the vision system installed onto the rover and will be transmitted to a ground station.

\subsection{Robotic Rover Design}

Each mechanical parts were designed by using 3D modeling software where the rover consisted of a robotic arm, gripper, track, chassis, vision system and controller, as shown in Figure 1.

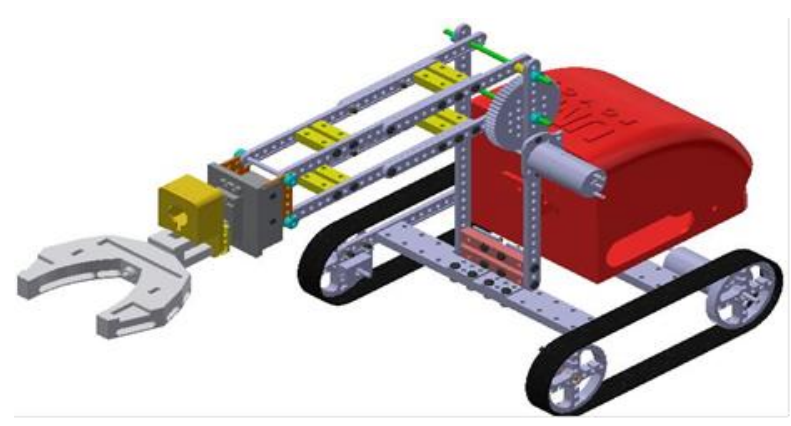

Figure 1. The design of the proposed robotic rover

\subsection{Design of the Arm and Gripper}

The arm and gripper of the rover were also designed using 3D modeling software [21] with the simplest design compared to the existing rover arms. Figure 2 shows the rover uses the gear as a moving mechanism of the arm by connecting it with the DC motor, where for motion transfer efficiency, the gear is equipped with interlocking teeth on its periphery (outer edge). 


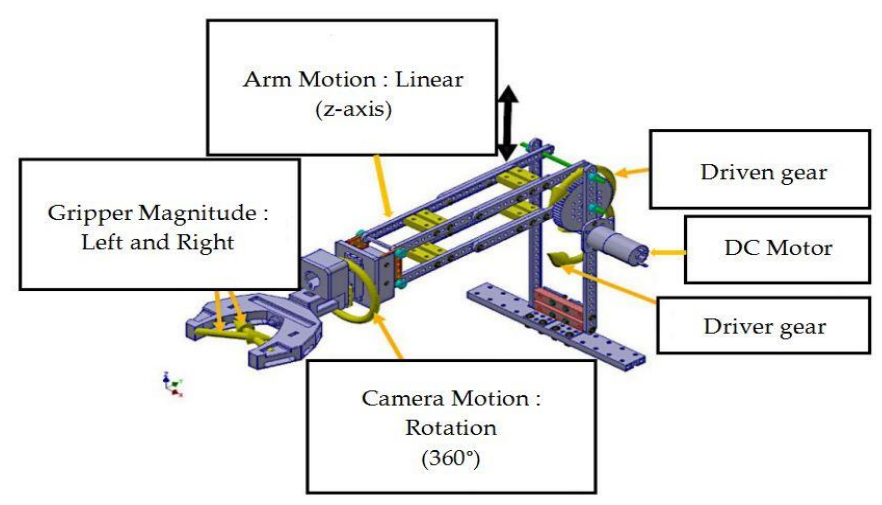

Figure 2. The design of arm and gripper

\subsection{Mobility System}

The robotic rover used a continues track that can travel along the terrain surfaces [22]. However, the current track is not suitable to be used on the smooth surface, so the new multifunctional track will be developed for the robotic rover to ease its mobility in all surfaces.

\subsection{Vision System}

A small camera was mounted on top of the arm gripper to enable First Person View (FPV) characteristics [23]. A monitor with receiver then converted the transmitter signals into images where the user can view real-time monitoring of the safety rover. Figure 3 shows the transmitter used in the rover and receiver system flowchart, respectively.

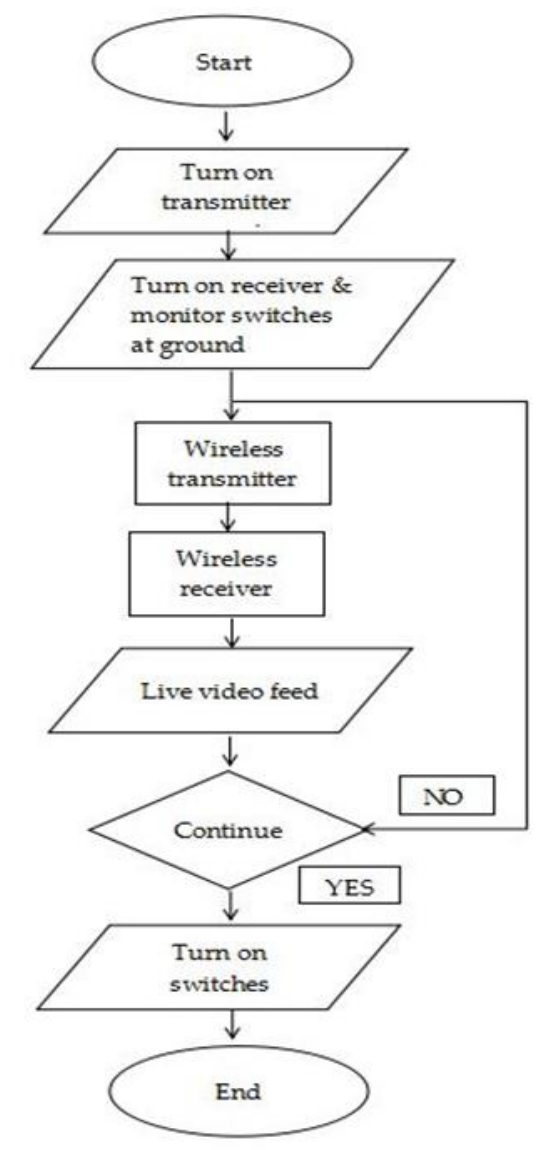

Figure 3. Flowchart of First Person View (FPV) system 


\subsection{Ground Station}

Figure 4 shows the completed setup of the ground station which consists of a receiver, LiPO Battery, remote control for the monitor, wireless joystick, switches and a 7-inch monitor. This station was used as storage for components required by the rover as well as the rover's movement monitoring kit.

Figure 5 shows a transmitter setup of FPV at a remotely controlled safety rover. The FPV camera was mounted with gripper while the transmitter and LiPO battery is connected in the wiring box with push-button.

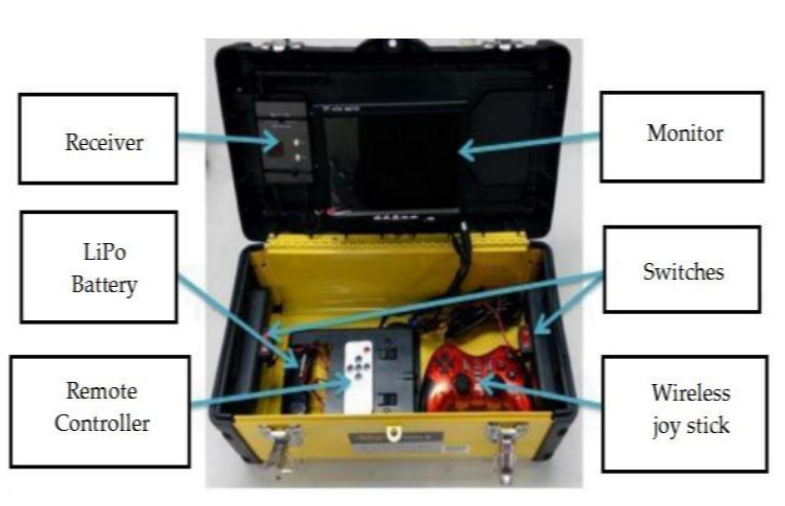

Figure 4. Ground station of the robotic rover

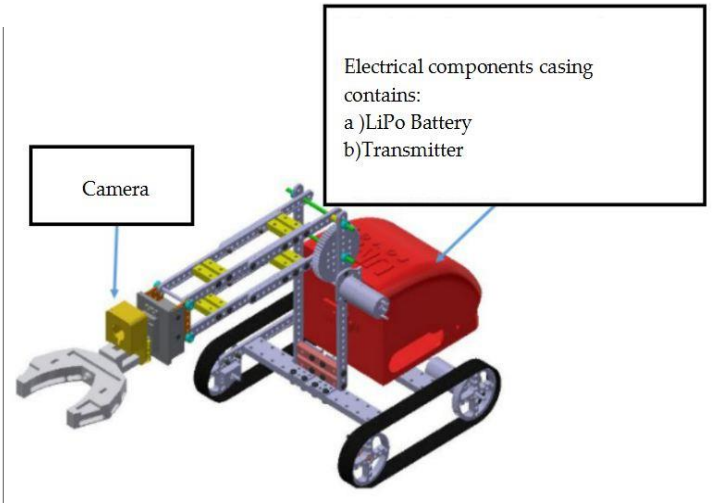

Figure 5. Transmitter setup at robotic rover

\subsection{Controller System}

In this application, encoder motors were used in tracks, servo motor was used in the robotic arm while a DC motor was used in the gripper. To control all these motors, joypad was used which acts as a remote controller.

This rover is controlled by the wireless controller via the USB host, where the receiver of this wireless controller is inserted in the female port of the USB host. The USB host is connected to the microcontroller, and to control this microcontroller, a wireless joystick is used, in which its receiver is connected to the microcontroller via the USB host.

\section{RESULTS AND DISCUSSIONS}

\subsection{Movement of the Rover}

With this dedicated code, a button on the controller joypad can be assigned to perform a task. For the left buttons and left joystick, these four buttons can be assigned to move the rover, which is forward, reverse, turn right and turn left. Figure 6 shows the movement of both wheeled motors, during programming.

Table 1 explains the directions of both wheeled motors, Motor 1 (M1) and Motor 2 (M2). In order to move the rover forward and reverse, both motors need to be alternated in directions. The motors for these forward and reverse motions are set to $180 \mathrm{rpm}$ (rotation per minute) where the maximum speed of these motors is $185 \mathrm{rpm}$.

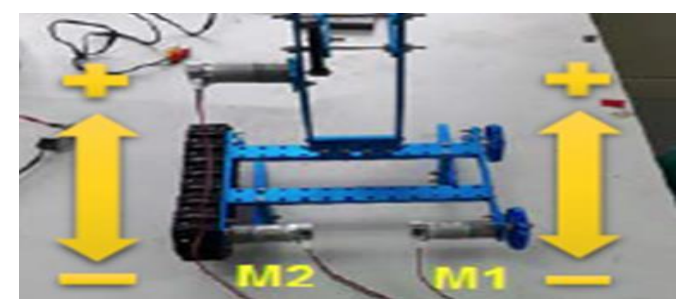

Figure 6. The movement of the wheeled motors

The movements of the robot arm, the gripper and the servo are shown in Figure 7(a) and 7(b). The rover arm is programmed to move upwards and downwards. To move this robotic arm, the DC motors 
with an encoder are used where the maximum speed of this motor is $86 \mathrm{rpm}$. The rover arm is attached with the gear and this gear is being rotated by the motor to move the arm.

Table 1. The Direction of Both Wheeled Motors

\begin{tabular}{cccc}
\hline Rover & Motor 1 (M1) & Motor 2 (M2) & Explanation \\
\hline Turn Right & CW $(-)$ & CW $(-)$ & Both motors in the same clockwise (CW) direction \\
Tern Left & CCW $(+)$ & CCW $(+)$ & Both motors in the same counter-clockwise direction \\
Forward & CCW $(+)$ & CW $(-)$ & The motors need to be in alternate directions \\
Reverse & CW $(-)$ & CCW $(+)$ & The motors need to be in alternate directions \\
\hline
\end{tabular}

In Figure 7(b), the servo is programmed to rotate in clockwise (CW) and counter-clockwise (CCW) directions. The type of the servo is a continuous rotation servo. To program this motor to stop, the $90^{\circ}$ angle command is required whereas to program this motor to rotate in counter-clockwise, coding it with an angle more than $90^{\circ}$ is acquired, and in clockwise direction, coding it with an angle less than $90^{\circ}$ is acquired. For the gripper, the normal DC motor is being used which rotates it. On the other hand, to rotate it in a counter direction, the polarity has to be changed.

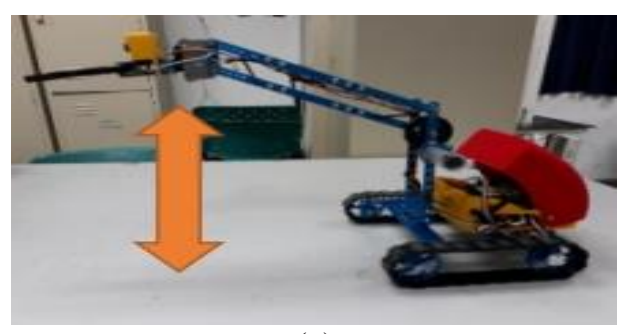

(a)

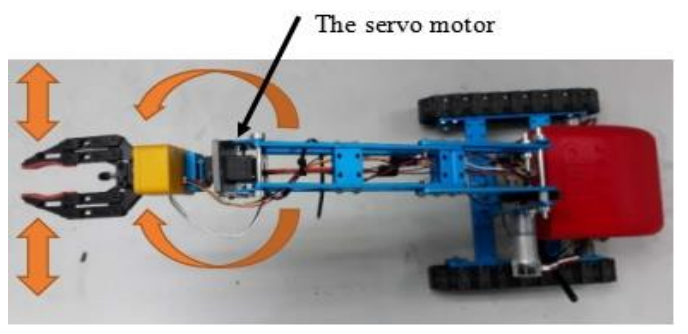

(b)

Figure 7. The movement of the rover (a) robotic arm, and (b) servo motor and gripper

\subsection{Performance of the Rover Arm}

The specification of the developed rover is explained in Table 2 whereas the conditions of maximum and minimum height of the rover arm are shown in Figure 8. The analysis had been conducted on its ability to lift object and the results stated that the lightest object it can lift is a marker whereas the heaviest it can load is such as the small mosquito's spray weighing around 750 gram, as shown in Figure 9.

Table 2. Specification of Developed Robotic Rover

\begin{tabular}{cc}
\hline Specification & Detail \\
\hline Rover weight & $2.2 \mathrm{~kg}$ \\
Weight of ground station & $3.0 \mathrm{~kg}$ \\
Maximum arm height & 0.483 meters \\
Minimum arm height & 0.013 meters \\
\hline
\end{tabular}

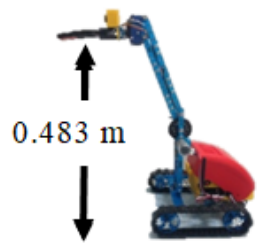

Figure 8. Maximum and minimum distance of arm lift
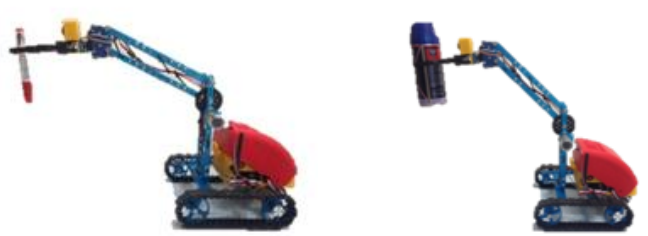

Figure 9. Capabilities of rover to lift objects

The result shows the rover has limitations to lift objects. It cannot lift an object more than 750 gram where it will start to collapse. Currently, the arm can only move in a transverse motion where the rover needs to be modified by adding an additional degree of freedom to make it more stable. 


\subsection{Performance of Vision System}

Probably the biggest problem in remotely working with any vehicle is giving the administrator an easy-to-understand, natural approach to control it. A powerful method for managing this issue is by giving a vision-based interface [24]. Through an onboard point of view, the user can better comprehend the environment and receive more instinctive input of the position and the condition of the remotely operated vehicle (ROV) [25]. The first-person view (FPV) is a guide utilized by the pilots to encourage control of the remote-controlled vehicle through its perspective. In this manner, the FPV camera is utilized to capture the picture and deliver it to the transmitter which will then produce a wireless video signal and transmits it to the ground utilizing an antenna. At that point, the FPV video receiver (VRX) is utilized to obtain a wireless video signal using a specific antenna and transmits the signal to a viewing device such as a monitor screen or an FPV goggle.

This study successfully installed the vision system on the rover and received wireless from the ground station the as shown in Figure 10. The receiver on the ground station could capture the vision of the rover movement as far as 20 meters.

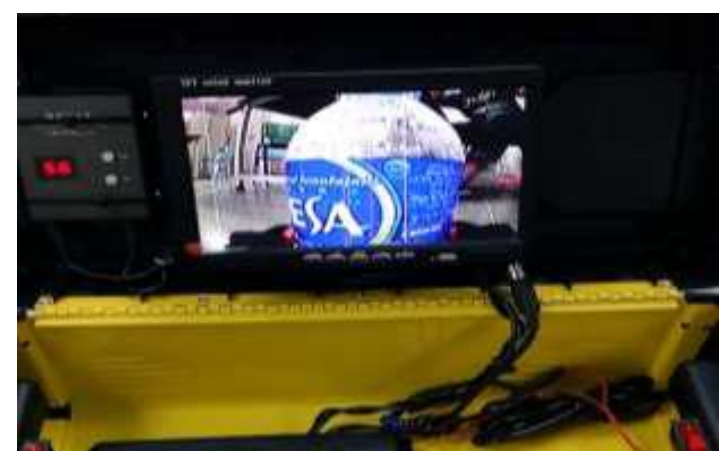

Figure 10. Rover vision system performance on the ground station

\subsection{Performance feedback}

Data were collected from the members of Hazard Material (HAZMAT). As shown in Figure 11, they tested our rover and were satisfied with this invention because their department required a technology equipment as safety precautions for them to handle dangerous situations [26]. From their opinion, this rover is suitable for Chemical Biological Radiation Nuclear explosive (CBRNe) unit [27], which is a special unit that deals with chemical weapon to assist them do sampling pump by using toxic gas assessment [28] test kit (GASTEC), that conduct surveys to detect particles and radiations as well as samplings [29] for explosive substances from far.

Survey was conducted at two Fire Departments, Bandar Baru Bangi and Shah Alam (HAZMAT), and received responses from around 30 respondents. Based on the result shown in Figure 12, it can be concluded that the robotic rover is suitable for dangerous and contaminated areas [30].

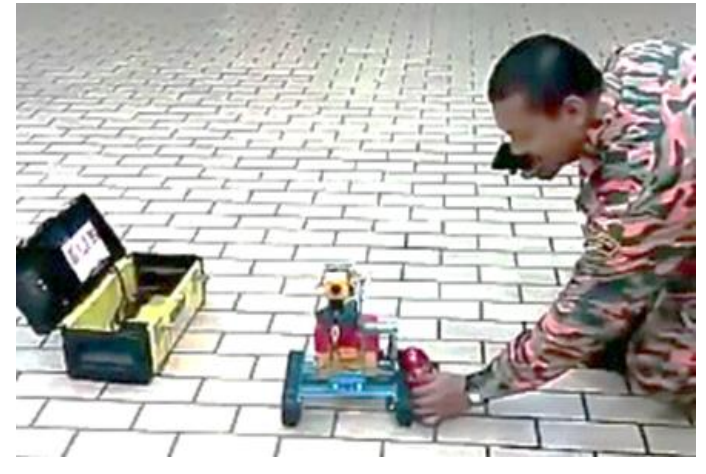

Figure 11. A member of HAZMAT unit is testing the performance of the robotic rover

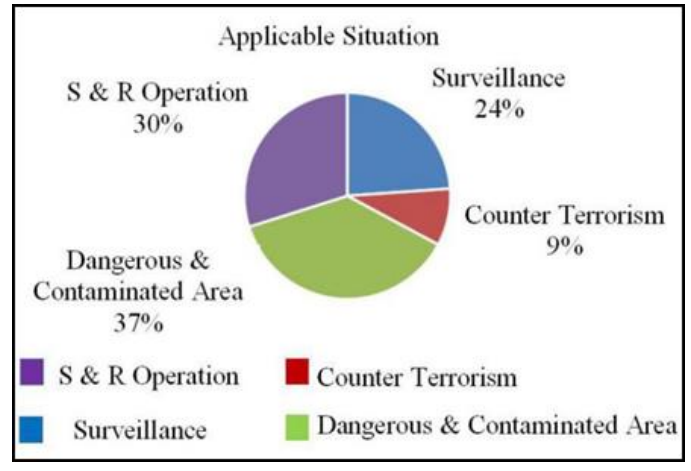

Figure 12. Result of the applicable situations 


\section{CONCLUSION}

In conclusion, the goal of this study is to produce a simple robotic rover with an easy-to-build system and manufacture its structure. There are three main objectives of the robotic rover which are designed for work lift, monitoring and safety which can be achieved when the robotic rover is equipped with the robotic arm and gripper to collect and remove load. In addition, it can be used to monitor dangerous situations during hazardous condition and the most crucial thing is, it can reduce or eliminate risk to personnel's life because it can be controlled by using vision and joystick controller.

For future recommendations, HAZMAT members recommended to include additional features to the robotic rover. First is the gas detector commonly used in the detection of toxic gases like Carbon Monoxide (CO), Oxygen (O2), Hydrogen Sulfide (H2S), and LEL (Lower Explosive Limit). The LEL detector identifies, shows and cautions for levels between $0-100 \%$ when the electrodes deliver signals if gas is detected. Second is the thermal imaging which is a strategy for improving the permeability of objects under a dark situation by distinguishing the items' infrared radiation and developing a picture dependent on that data. Lastly, the rover also needs a flashlight to light up the dark places to monitor the surroundings.

\section{ACKNOWLEDGEMENTS}

This project was funded by Automotive Excellence Centre, Universiti Malaysia Pahang using research grant RDU1903103. This work was also a collaborative work with the German Malaysian Institute under Senior Design Project course.

\section{REFERENCES}

[1] C. Shao, S. Tanaka, et al.” A Tactile Sensor Network System Using a Multiple Sensor Platform with a Dedicated CMOS-LSI for Robot Applications", Sensors 2017, 17, 1974.

[2] D. Miglani and A. Hensman, "Vision for secure home robots: Implementation of two-factor authentication" IEEE International Symposium on Technology and Society (ISTAS) 2015, Dublin, 11-12 November 2015.

[3] S. Ichael and W. Armin, "The LRU Rover for Autonomous Planetary Exploration and its Success in the Space Boot Camp Challenge, " International Conference on Autonomous Robot Systems and Competitions, pp.7-11, 2016.

[4] P. Lehner, et al., "Mobile manipulation for planetary exploration", IEEE Aerospace Conference 2018, USA, 3-10 March, 2018.

[5] C. Collins and M. Robinson, "Accuracy analysis and validation of the Mars Science Laboratory (MSL) robotic arm", Proceedings of The ASME 2013 International Design Engineering Technical Conferences, H. Poor, An Introduction to Signal Detection and Estimation. New York: Springer-Verlag, 2013, Ch. 4, 1985.

[6] Hemanth Reddy A, B. Kalyan, C. S. N. Murthy, "Mine Rescue Robot System - A Review”, Procedia Earth and Planetary Science, Vol. 11, pp. $457-462,2015$.

[7] A.B. Dzulqarnain and I. Norazura, "The Integral Approach to Safety,"in International Journal of Engineering Technology and Sciences (IJETS), Vol. 6, No.1, 2016.

[8] M. Steven, "The Rise of the Robots: What the Future Holds for the World's Armies", Fast Company, December 6, 2017.

[9] T. Cristen and K. Sara, " How a Robot Should Give Advice”, Proceedings of the Human Robot Interaction, pp. 275282, 2013.

[10] Y. Kobayashi, et al., “Assisted-care robot dealing with multiple requests in multi-party settings,” 6th ACM/IEEE International Conference on Human-Robot Interaction, March 2011, Switzerland.

[11] K. Perumal, M. A. M. Ali and Z. H. Yahya, "Fire Fighter Robot with Night Vision", IEEE 15th International Colloqium on Signal Processing and Its Application, March 2019, Penang.

[12] C. Tamar and A. Mark B, "Interactive 3D Visualization within Eclipse". NASA Ames Research Center, USA: NASA Production, 2014.

[13] C. Joy A. A. Mark, et al, "Mars Exploration Rover Mission," Journal of Geophysical Research. Vol. 108, Issue E12, 2018.

[14] K. Konstantin, "What Drones Can Do and How They Can Do It," Drones and Aerial Observation. New Technologies for Property Rights, Human Rights, and Global Development, July: 9-17, 2015.

[15] T-S. Chu, et al., "Perceiving intimacy from both robot view and first-person view in dyadic human interaction", IEEE International Workshop on Advanced Robotics and Its Social Impact 2014, 11-13 September 2014, USA.

[16] Gil. G, Savino, G. Piantini, S. Pierini, et al,". Motorcycles that See: Multifocal Stereo Vision Sensor for Advanced Safety Systems in Tilting Vehicles," Sensors 2018, 18, 295, 2018

[17] Gotovtsev, P. Vorobiev, V. Migalev, A. Badranova and et.al, "Based Power Sources for Mobile Autonomous Robots". Robotics, Vol. 7, No. 2, 2018.

[18] Adarsh R.S. and M. M. Dharmana, "Multi-terrain Multi-Utility Robot”, Procedia Computer Science, Vol. 133, pp. 651-659, 2018.

[19] W. Li, et al., "Semi-autonomous bilateral teleoperation of six-wheeled mobile robot on soft terrains", Mechanical System and Signal Processing, Vol. 133, 2019. doi.org/10.1016/j.ymssp.2019.07.015.

Indonesian J Elec Eng \& Comp Sci, Vol. 18, No. 2, May 2020 : 766 - 773 
[20] T. Guo, J. Guo, B. Huang, H. Peng, "Power consumption of tracked and wheeled small mobile robots on deformable terrains-model and experimental validation”, Mechanism and Machine Theory, Vol. 133, pp. 347 - 364. 2019.

[21] Sokolov, M., Lavrenov, R., Gabdullin, A., Afanasyev, I., \& Magid, E., 3D modelling and simulation of a crawler robot in ROS/Gazebo. In Proceedings of the 4th International Conference on Control, Mechatronics and Automation, pp. 61-65, 2016.

[22] Zong, C., Ji, Z., \& Yu, H., Dynamic stability analysis of a tracked mobile robot based on human-robot interaction. Assembly Automation. 2019.

[23] Xia, L., Gori, I., Aggarwal, J. K., \& Ryoo, M. S., Robot-centric activity recognition from first-person rgb-d videos. In 2015 IEEE Winter Conference on Applications of Computer Vision (pp. 357-364). 2015.

[24] Mohatta, S., Perla, R., Gupta, G., Hassan, E., \& Hebbalaguppe, R. Robust hand gestural interaction for smartphone based AR/VR applications. In 2017 IEEE Winter Conference on Applications of Computer Vision, pp. 330-335, 2017.

[25] Chu, Z., Zhu, D., \& Yang, S. X. Observer-based adaptive neural network trajectory tracking control for remotely operated vehicle. IEEE Transactions on Neural networks and learning systems, 28(7), 1633-1645, 2016.

[26] Schneider, Frank E., and Dennis Wildermuth. "Using robots for firefighters and first responders: Scenario specification and exemplary system description." In 2017 18th International Carpathian Control Conference (ICCC), pp. 216-221, 2017.

[27] Baums, A. Response to CBRNE and human-caused accidents by using land and air robots. Automatic Control and Computer Sciences, 51(6), 410-416, 2017.

[28] Wei, G., Gardner, J. W., Cole, M., \& Xing, Y. Multi-sensor module for a mobile robot operating in harsh environments. In 2016 IEEE SENSORS, pp. 1-3, 2016.

[29] Xing, Y., Vincent, T. A., Cole, M., Gardner, J. W., Fan, H., Bennetts, V. H., \& Lilienthal, A. J., Mobile robot multisensor unit for unsupervised gas discrimination in uncontrolled environments. In 2017 IEEE SENSORS, pp. 1-3, 2017.

[30] Kopuletý, Michal, and Tibor Palasiewicz. "Advanced military robots supporting engineer reconnaissance in military operations." In International Workshop on Modelling and Simulation for Autonomous Systems, pp. 285-302. Springer, Cham, 2017. 